\title{
CREATIVIDAD Y TECNOLOGÍAS EMERGENTES EN EDUCACIÓN
}

\author{
Yésica del Águila Ríos \\ Departamento de Didáctica \\ Universidad de Cádiz \\ yessica.delaguila@uca.es \\ Ma Regina Teixeira Ferreira Capelo \\ CLEPUL/Polo of Madeira \\ John Miguel Costa Varela \\ CLEPUL/Polo of Madeira \\ Jorge Guerra Antequera \\ Departamento Didáctica \\ Universidad de Extremadura \\ Juan Antonio Antequera Barroso \\ Departamento de Didáctica \\ Universidad de Cádiz
}

Fecha de Recepción: 28 Enero 2019

Fecha de Admisión: 30 Abril 2019

\section{RESUMEN}

La creatividad es un valor en alza debido a su estrecha relación con la solución de problemas y el emprendimiento. Es conveniente que esta creatividad sea fomentada desde los primeros años de escolarización cuando el mundo de fantasía y simbólico del niño está en plena ebullición y dónde todo es posible. Así, el fomento de la creatividad, aparece convenientemente contemplado en el currículum de la etapa.

Por otro lado, desde las administraciones educativas se está promoviendo el uso de metodologías didácticas en las que el alumnado, como protagonista de su aprendizaje, participa de forma activa en la creación de contenidos y materiales educativos. Este nuevo escenario está generando nuevos retos y aprendizajes para los docentes. Se hace imprescindible conocer y dominar qué herramientas y estrategias son necesarias en el mundo de la información y conocimiento. El profesorado ya no puede seguir siendo el mismo que en el pasado dado que desempeña otra tarea muy distinta: la de enseñar a aprender con los medios que están al alcance de su alumnado y no enseñar contenidos como antaño.

Los resultados consistentes con la literatura hacen imposible concebir un currículo que promueva la creatividad sin educación para los medios y sin las tecnologías emergentes que se puedan aplicar en el aula.

Palabras clave: tecnologías emergentes; creatividad; educación; metodologías didácticas 


\section{CREATIVIDAD Y TECNOLOGÍAS EMERGENTES EN EDUCACIÓN}

\section{ABSTRACT}

Creativity and emerging technologies in education. Creativity is a rising value due to its close relationship with problem solving and entrepreneurship. It is convenient that this creativity be fostered from the first years of schooling when the fantasy and symbolic world of the child is in full boiling and where everything is possible. Thus, the promotion of creativity is conveniently contemplated in the curriculum of the stage.

On the other hand, the educational administrations are promoting the use of didactic methodologies in which the students, as protagonists of their learning, participate actively in the creation of content and educational materials. This new scenario is generating new challenges and learning for teachers. It is essential to know and master what tools and strategies are necessary in the world of information and knowledge. The teaching staff can no longer be the same as in the past given that they perform another very different task: that of teaching to learn with the means available to their students and not teaching content as in the past.

The results consistent with the literature make it impossible to conceive a curriculum that promotes creativity without education for the media and without the emerging technologies that can be applied in the classroom.

Keywords: emerging technologies; creativity; infant education; didactic methodologies

\section{INTRODUCCIÓN}

Desde las administraciones educativas se está promoviendo el uso de metodologías didácticas en las que el alumnado, como protagonista de su aprendizaje, participe de forma activa en la creación de contenidos y materiales educativos. De esta forma, se pueden obtener resultados significativos con respecto a la adquisición de contenidos y a la creación de situaciones de enseñanza directamente relacionadas con la vida real de los "nativos digitales" (Prensky, 2011). La implicación del alumno en la escuela y en los aprendizajes, debe estar ligada la experiencia de los estudiantes (González, 2010). En este sentido, es importante entender que el alumnado se encuentra en un momento ideal para conocer y dominar las habilidades de aprendizaje necesarias que le permitan adquirir conocimientos directamente relacionados con el mundo virtual en el que se encuentra inmerso. Por tanto, como docentes debemos plantear propuestas educativas que promuevan su pensamiento divergente y, a la vez, desarrollen rutinas de pensamiento que le ayuden a pensar, a resolver problemas, a investigar, a seleccionar contenidos y materiales, a tomar decisiones, trabajar en equipo, valorar trabajos y producciones propios y de compañeros (Domínguez y Pineda, 2019).

Este nuevo escenario "de sabiduría de masas, de inteligencia colectiva" (Delclós, Tomàs, 2009), "de alquimia de multitudes" (Pisani y Piodet, 2009) o "tecnologías emergentes" (Adell y Castañeda, 2012) está generando nuevos retos y aprendizajes para los docentes en el marco educativo actual. Es necesario entender y dominar las habilidades y competencias necesarias que permitan utilizar de manera eficaz las herramientas y estrategias adecuadas para el intercambio positivo de información (Gómez y Cano, 2011). En consecuencia, el docente debe "derrochar imaginación e inventar... es necesario adaptar los materiales a la "Iengua" de los Nativos" (Prensky, 2010, p.9). Por otra lado, Buckingham (2005) establece como principal prioridad conocer qué capacidades y/o actitudes se ponen en juego durante su utilización ya que contribuirá de manera significativa a "la formación de nuestra sabiduría, para poder tomar decisiones y juicios más acertados” (Prensky, 2010, p.94). En la misma línea, Comas, Sureda, Casero y Morey (2011) desarrollan la tesis de Buckingham (2005) y Prensky (2010) insiste en la idoneidad de realizar propuestas educativas que analicen los nuevos usos y posibles dificultades que se estén produciendo.

En la actualidad, la inclusión en el aula de las metodologías emergentes supone una re-confi- 
guración y recontextualización de los procesos de enseñanza y de aprendizaje (Sosa, 2019) ya que "son sin duda el principal recurso contemporáneo de expresión y comunicación cultural: quien pretender participar activamente en la vida pública necesariamente tendrá que utilizar los modernos medios de comunicación social" (Buckinghan, 2005: p.22). En este sentido, Campos y Palacios (2018, p. 179) resaltan que el fomento del proceso creador en las instituciones educativas no está siendo una tarea fácil ya que no pueden seguir educando en el pasado. Este panorama representa "un desafío para el contexto escolar en general y los maestros, ya que significa repensar objetivos, técnicas de enseñanza, formas de evaluar y por sobre todas las cosas la renovación auténtica de los procesos de enseñanza -aprendizaje. Solo de este modo, se podrá dar el salto a la educación del siglo XXI".

Algunas metodologías que podemos poner en práctica para generar contenidos de aprendizajes significativos y válidos son: la gamificación, los entornos personales de aprendizaje, design thinking, pedagogía inversa o flipped classroom, aprender a emprender, realidad aumentada, rutinas de pensamiento y aprendizaje cooperativo.

En primer lugar, la gamificación se define como "la utilización de mecanismos, la estética y el uso del pensamiento, para atraer a las personas, resolver problemas, promover la acción y el aprendizaje" (Kapp, 2012, p.9). En otras palabras, se utilizan la características y estructuras de los juegos en contextos de aprendizaje no lúdicos. Sánchez y Rivas (2015) recopilan un total de seis características propias que se encuentran en la mayoría de los proyectos gamificados, entre ellas; un aumento de la motivación gracias a la creación de propuestas educativas acordes al nivel competencial del alumnado, resultados visibles durante el proceso de participación, la retroalimentación constante que genera, los distintos niveles de complejidad que ofrece, la evaluación de procesos y de resultados y la autonomía que se genera en la decisión y toma de decisiones.

Adell y Castañeda (2010, p.10) conciben estos entornos de aprendizajes "como el conjunto de herramientas, fuentes de información, conexiones y actividades que cada persona utiliza de forma asidua para aprender". Es decir, se refieren tanto a los recursos que se seleccionan para acceder a la información como a las relaciones que se establecen durante este proceso y personas que ofrecen asesoramiento y guía. Por tanto, son necesarias las herramientas y recursos que nos aportan la Web 2.0 y las estrategias multimedias que se usan para su manejo.

En segundo lugar, se encuentra otra metodología didáctica denominada design thinking (Sánchez, Torres y Romina, 2015) que ofrece una oportunidad única para la creación de pensamiento divergente en donde el alumnado plantea el problemas y los recursos y medios necesarios para su resolución.

En tercer lugar, clase invertida o flipped classroom se define "como un modelo pedagógico que se basa en la inversión de la estructura tradicional de la clase presencial expositiva a través del empleo de tecnologías de información y comunicación" (Olaisola, 2014, p.1). De esta forma, el alumnado es quien dirige, crea y gestiona su propio aprendizaje a partir de las premisas e indicaciones ofrecidas por el docente.

En definitiva, el uso de metodologías activas en el aula aumenta el compromiso y motivación del alumnado por la enseñanza y aprendizaje (Learreta, Montil, González y Asensio, 2009). Asimismo, 0 Reilly (2004) sostiene que los nuevos avances, científicos y tecnológicos llevan consigo nuevos usos y posibilidades para los usuarios. Por tanto, los usuarios y las usuarias digitales (Augé, 1992 y Aparici, 2010) tienen una gran facilidad para adquirir e intercambiar cualquier tipo de información y conocimiento. Así es la Web del momento: "se intercambian información, se hacen comentarios, se implican, participan, etc. ya no se contentan con navegar, con surfear. Actúan." (Pisani y Piodet, 2009: p.14). De esta forma, todos son emisores -activos creadores- y receptores, a la vez que se 


\section{CREATIVIDAD Y TECNOLOGÍAS EMERGENTES EN EDUCACIÓN}

ponen en juego nuevos medios de comunicación caracterizados por la interactividad y la actualización constante en tiempo real. Para Rheingold (2004: p.142), "el intercambio de conocimientos no es lo novedoso [...]. En cambio, el intercambio en tiempo real no sólo es novedoso, sino que transforma radicalmente el proceso comunicativo".

No existen dudas que la inclusión de estas metodologías emergentes y activas en el currículo educativo conlleva un cambio en el planteamiento didáctico pedagógico de qué, cómo y cuándo enseñar y qué, cómo y cuándo evaluar. Por todo ello, es necesario una enseñanza que promueva el desarrollo de la capacidad creativa. Nos encontramos ante la necesidad absoluta de promover una educación para el pensamiento creativo"el proceso donde los docentes tenemos que actuar como guías en el camino hacia el mundo del mañana que forjarán nuestros estudiantes” (Sumo, Voisin y Téllez, 2016, p. 87).

Los principales factores o indicadores criteriales básicos que aportan información sólida y valiosa sobre el estilo y el potencial creativo de las personas y que pueden condicionar su desarrollo (De Prado, 1998, pp. 7-10 y Núñez-Lagos, 2006, pp. 87-88):

- La originalidad: Hace referencia a lo nuevo y único, a lo avanzado y pionero. Es el mejor rasgo que resume la creatividad.

- La fluidez y productividad: Se refiere a la cantidad de soluciones y respuestas dadas por el sujeto ante una situación. Junto con la flexibilidad y originalidad forman parte de los criterios de diagnósticos de la creatividad.

- La flexibilidad: Es lo opuesto a la rigidez. La creatividad es agilidad mental, es decir, tener múltiples perspectivas distintas y contrarias.

- La elaboración: Se relaciona con el procesamiento y organización de la información, valorándose positivamente la capacidad de las personas para expresarse y aclarar las cosas con la mayor precisión posible.

- El análisis: Es la capacidad que permite descomponer mentalmente un hecho en sus partes y comprenderlo.

- La síntesis: Está relacionada con la capacidad de elaborar esquemas, organizar la información y extraer los rasgos más valiosos generando una nueva teoría o composición original.

- La sensibilidad para los problemas: Es la empatía para percibir y descubrir situaciones peligrosas, difíciles y problemáticas. Cada persona trata de encontrar las mejores soluciones posibles.

- La apertura mental: Hace referencia a la aptitud vital de las personas para aceptar nuevas experiencias y sorprenderse gratamente y afrontar con serenidad retos y obstáculos, buscando la mayor cantidad de alternativas posibles de resolución.

- La comunicación: Es la capacidad de transmitir y compartir mensajes, productos y descubrimientos con otras personas. Está ligada a las capacidades expresivos emotivo gestuales y verbales delas personas.

- La redefinición: Es la capacidad de encontrar usos, funciones, aplicaciones y definiciones diferentes a las habituales.

- La inventiva: La habilidad para percibir la realidad del modo nuevo y transformarla parcial 0 totalmente. La creatividad es inventiva, sé inventor en cada acción o ante cada objeto. Cámbialo hasta que no se parezca en nada al original.

Estos factores ofrecen información relevante sobre el concepto de creatividad y han de ser puestos en práctica en el aula de educación infantil si queremos ofrecer a nuestro alumnado una enseñanza realmente creativa e inventiva (Franco, 2004).

Dichos aspectos se sustentan en los trabajos realizados por Lillian y Logan, 1980 (cit. por Borthwick, 1982, pp. 55-59) quienes destacaron los aspectos que se debían tener en cuenta para 
fomentar la creación creativa en los alumnos: flexibilizar la enseñanza, prestando atención a las diferentes capacidades, intereses e historia personal y escolar del niño; las actividades propuestas deben estar interrelacionadas y forman un conjunto; utilización de métodos indirectos de enseñanza; aquí la enseñanza se convierte en un arte, el arte de deducir, preguntar, sugerir, proporcionar pistas, indicar alternativas e integrar; las tareas de enseñanza deben estimular respuestas creativas tanto para los docentes como para los alumnos; el maestro imaginativo utiliza experiencias anteriores, combina materiales, métodos, ideas y medios de formas nuevas existentes, que ayudan a que los niños se integren en la enseñanza y refuercen los conceptos; fomenta el uso único de materiales e ideas; el maestro debe conocer tanto los materiales para animar a los alumnos a jugar con las ideas, como los sistemas para modificar el medio de enseñanza a fin de facilitar un comportamiento creativo por parte de los alumnos; implica una interacción en la que intervienen el profesor, el alumno, el tema y una experiencia o actividad de aprendizaje particular; el maestro enseña, pero es el alumno el que aprende; crea el ambiente en el que se fomenta la curiosidad, investigación y la experimentación; implica autovaloración y representa un importante papel en la enseñanza y el aprendizaje creativo.

Para una valoración y comprensión de este proceso creador se propone el diseño de actividades sencillas, concretas y fáciles de aplicar en esta etapa educativa. Para ello, proponemos la utilización de activadores creativos que son "estimuladores eficaces para desencadenar de modo fácil y operativo diversos procesos y actividades divergentes, innovadores e inventivas, que de un modo nuevo y original fomentan la utilización de todo el cerebro" (De Prado, 1996, p. 6). Pawlak (2000) defiende que estas herramientas, que promueven una educación creativa, suponen también un ambiente estimulante, líderes naturales y equipos de trabajo multidisciplinarios. Por su parte, Núñez y Lagos (2006, p. 90) aportan las características que debe poseer un docente para orientar correctamente a sus alumnos: tener una alta motivación intrínseca hacia el trabajo que realiza, ser flexible y poseer una independencia de pensamiento, junto con la posición activa y transformadora, poseer una autoconciencia ya autovaloración. Estos valores le permiten variar una situación en función de las necesidades, propicia la divergencia y acepta las críticas, valora al alumno y le facilita los medios y estímulos para que desarrolle sus propios recursos, se promueve el aprendizaje por descubrimiento, es laborioso al despertar gran curiosidad por todo lo que le rodea, hace un uso racional de su autoridad, aprovecha cualquier situación, cualquier recurso, permite la libertad de movimientos y favorece la realización de experiencias.

En este modelo de enseñanza, el maestro interacciona y se acerca al alumnado, el verdadero protagonista del proceso de enseñanza y aprendizaje, pues de lo contrario "a no ser que dispongamos de maestros creativos, los alumnos que se atrevan a crear, imaginar y cuestionar, seguirán siendo marginados por maestros hostiles" (Torrance, 1961, p. 23).

Para finalizar, consideramos importante realizar una revisión sobre el tratamiento normativo de la creatividad en nuestro País. Concretamente, en la Ley Orgánica 2/2006, de 3 de mayo de Educación (LOE, texto consolidado, 2018), en su artículo 2 "fines de la educación" se recoge que "f) El desarrollo de la capacidad de los alumnos para regular su propio aprendizaje, confiar en sus aptitudes y conocimientos, así como para desarrollar la creatividad, la iniciativa personal y el espíritu emprendedor" (p.15). En contraposición, encontramos que en el artículo 12 sobre "principios generales de la Educación Infantil" no se hace mención a la capacidad creativa si no que se trata aspectos generales de la etapa. También encontramos que en la Ley 8/2013, de 9 de diciembre, para la Mejora de la Calidad Educativa (LOMCE) en su preámbulo (IV) se expresa que "es necesario adquirir desde edades tempranas competencias transversales, como el pensamiento crítico, la gestión de la diversidad, la creatividad o la capacidad de comunicar..." (p.97860). 


\section{CREATIVIDAD Y TECNOLOGÍAS EMERGENTES EN EDUCACIÓN}

Por su parte, el Real Decreto 1630/2006, de 29 de diciembre, por el que se establecen las enseñanzas mínimas del segundo ciclo de Educación Infantil, indica que "el currículo se orienta a lograr un desarrollo integral y armónico de la persona en los distintos planos: físico, motórico, emocional, afectivo, social y cognitivo" (p.474) pero no se hace mención directa a la creatividad. Sin embargo, en el área "Lenguajes: Comunicación y representación". del Segundo Ciclo de Educación Infantil se menciona que "El lenguaje artístico hace referencia tanto al plástico como al musical. El lenguaje plástico tiene un sentido educativo que incluye la manipulación de materiales, texturas, objetos e instrumentos, y el acercamiento a las producciones plásticas con espontaneidad expresiva, para estimular la adquisición de nuevas habilidades y destrezas y despertar la sensibilidad estética y la creatividad" (p. 480).

Igualmente, en el Decreto 428/2008, de 29 de julio, por el que se establece la ordenación y las enseñanzas correspondientes a la Educación Infantil en Andalucía que aunque tampoco aparece el término de creatividad sí se incluye en el artículo 6, sobre orientaciones metodológicas, referencias a la importancia de atender a las conductas exploratorias y la indagación en Educación Infantil "partir de los conocimientos previos, necesidades y motivaciones de cada niño o niña, propiciar la participación activa de éstos, fomentar sus aportaciones, estimular el desarrollo de sus potencialidades y facilitar su interacción con personas adultas, con los iguales y con el medio" (p. 9).

Finalmente, en la Orden de 5 de agosto de 2008, por la que se desarrolla el Currículo correspondiente a la Educación Infantil en Andalucía, encontramos en varias ocasiones referencias a la promoción de situaciones educativas y al uso de distintos recursos materiales que potencien la creatividad. De igual forma, se considera al tutor como persona responsable para promover esta creatividad "en los niños y niñas en sus producciones plásticas, tanto en situaciones libres como dirigidas, estableciendo a veces algunas condiciones para la producción. Condiciones en todo caso destinadas a potenciar el desarrollo de su pensamiento divergente" (p. 45).

En conclusión, la revisión bibliográfica y legislativa presentada pone de manifiesto la idoneidad de realizar propuestas educativas que promuevan el uso de estrategias didácticas colaborativas y significativas para el desarrollo del pensamiento divergente. Por tanto, se propone la planificación de secuencias de actividades sencillas, significativas, prácticas y reales que cuyo objetivo principal sea la participación activa del alumnado como principal protagonista en este proceso de enseñanza y aprendizaje. En esta apuesta, cobra especial significación el trabajo en equipo y la resolución de problemas cercanos a la vida real de los alumnos y las alumnas; de ahí la importancia de conectar con sus intereses, motivaciones y necesidades.

\section{REFERENCIAS BIBLIOGRÁFICAS}

Adell, J. y Castañeda, L. (2012). Tecnologías Emergentes, ¿Pedagogías Emergentes?. En Hernádez, M., Pennesi, M., Sobrino, D. y Vázquez, A. (coord.), tendencias en emergentes en educación con TIC. Asociación Espiral, Eduación y Tecnología, pp.14-32. Barcelona

Adell, J. y Castañeda, L. (2010). Los Entornos Personales de Aprendizaje (PLEs): Una nueva manera de entender el aprendizaje. En Roig, R. y Fiorucci, M. (Eds.), claves para la investigación en innovación y calidad educativas. La integración de las Tecnologías de la Información y la Comunicación y la Interculturalidad en las aulas. Alcoy: Marfil, Roma: TRE Universita degli studi.

Aparici, R. (coord.) (2010). Conectados en el Ciberespacio. Madrid: Universidad Nacional de Educación a Distancia.

Auge, M. (1992). Los no-lugares. Espacios del anonimato. Una antropología de la sobre modernidad. Barcelona: Editorial Gedisa.

Borthwick, G. (1982). Hacia una Educación Creativa. Madrid: Editorial Fundamentos. 
Buckingham, D. (2005). Educación en medios: alfabetización, aprendizaje y cultura contemporánea. Barcelona: Paidós comunicación 158.

Campos, G. y Palacios, A. (2018). La Creatividad y sus componentes. Creatividad y Sociedad, no 27.

Comas, R., Sureda, J., Casero, A., y Morey, M. (2011). La integridad académica entre el alumnado universitario español. Estudios Pedagógicos, 1, pp. 207-225.

Delclós, T. (2009). Las Ciudades Digitales Análisis del Estado de Digitalización de la Administraciones Públicas Madrileñas. En actas № A3: I Congreso Internacional Ciudades de Cultura. Madrid.

Domínguez, I. y Pineda, J, A. (2019). Arte y creación en el aula de Infantil. Investigación en la escuela, 97, pp. 21.

Franco, C. (2004). Aplicación de un programa psicoeducativo para fomentar la creatividad en la etapa de educación infantil. Relieve, 10 (2), pp. 245-266.

Gómez, J. y Cano, J. (2011). El pensamiento docente y su influencia en la implantación de las tecnologías de la información y la comunicación en el aula: Desafíos y oportunidades. Contextos Educativos, 14, pp.67-83.

González, M. T. (2010). El alumno ante la escuela y su propio aprendizaje: Algunas líneas de investigación en torno al concepto de implicación. REICE. Revista Iberoamericana sobre Calidad, Eficacia y Cambio en Educación, 8 (4), pp. 10-31.

Kapp, K. (2012). The Gamification of Learning and Instruction: Game-Based Methods and Strategies for Training and Education. San Francisco: John Wiley \& Sons.

Morales, F. M., Pérez, J. M., García, B. y Morales, F. M. (2017). Relación entre metodologías preferidas por estudiantes de grado y otras variables psicoeducativas. European Journal of Child Development, Education and Psychopathology 2017, 5, (2), pp. 72.

Núñez, I. y Lagos, B. (2006). La Creatividad en Educación Infantil. Revista Iberoamericana de psicomotricidad y técnicas corporales, 22, pp. 87-92.

Olaisola, A. (2014). La clase invertida: usar las tic para "dar la vuelta" a la clase. Facultad de Diseño y Comunicación - Universidad de Palermo.

0 Reilly, T. (2004). Whats Is Web 2.0. Design Patterns and Business Models for the Next Generation of Software. 0 reilly Network.

Pawlak, A. (2000). Fostering creativity in the new millennium. Research Technology Management, 43 (6), pp. 32-35.

Pisani, F. y Pionet, D. (2009). La Alquimia de las Multitudes. Cómo la Web está cambiando el Mundo. Barcelona: Ediciones Paidós Ibérica S. A.

Prado, D. (1998). 10 activadores creativos. Santiago de Compostela: Servicio de publicaciones e intercambio científico.

Prensky, M. (2010). Homo Sapiens Digital: De los Inmigrantes y Nativos Digitales a la Sabiduría Digital. En APARICI, R. (coord.), Conectados en el Ciberespacio (93-106). Madrid: Universidad Nacional de Educación a Distancia.

Prensky, M. (2011). Nativos e Inmigrantes Digitales. Institución Educativa SEK.

Rheingold, H. (2004). Multitudes inteligentes. La próxima revolución social. Barcelona: Gedisa Editorial.

Sánchez, E. y Pareja, D. (2015). La gamificación como estrategia pedagógica en el contexto escolar. En Ruiz, J., Sánchez-Rodríguez, J. y Sánchez-Rivas, E. (Edit.), innovaciones con tecnologías emergentes. Málaga: Universidad de Málaga.

Sánchez, M., Torres, B. y Díaz, R. (2015). Design thinking en las aulas. Cuadernos de Pedagogía, 453, pp. 34-37. 
Sosa, E. A. (2018). Diseño de un modelo de incorporación de tecnologías emergentes en el aula para la generación de estrategias didácticas por parte de los docentes. Universidad de Baleares.

Summo, V., Voisin, S. y Téllez, B. (2016). Creatividad: eje de la educación del siglo XXI. Revista Iberoamericana de Educación Superior, VII (18), 2016, pp. 83-98.

Torrance, E. (1961). Educación y capacidad crreativa. Madrid: Morata. 